\title{
Chameleon stars supported by a cosmological scalar field
}

\author{
Vladimir Folomeev * \\ Institute of Physicotechnical Problems and Material Science of the NAS of the Kyrgyz Republic, \\ 265 a, Chui Street, Bishkek, 720071, Kyrgyzstan
}

\begin{abstract}
Starting from the assumption that the present accelerated expansion of the Universe is driven by a chameleon scalar field, the function describing a direct coupling between the matter content of the Universe and the scalar field is derived from cosmological considerations. In progressing from cosmology towards astrophysical scales, this function is used to estimate the influence that such nonminimal coupling may have on the properties of usual (polytropic) stars whose matter can interact directly with the cosmological chameleon. It is shown that in order to obtain sizes and masses of compact configurations supported by the chameleon scalar field comparable to those of usual stars, it is necessary to assume that the pressure of the matter content of the Universe is substantially different from zero. The question of a possible manifestation of the effects of the nonminimal coupling in laboratory experiments is also discussed.

PACS numbers: 95.36. $+\mathrm{x}, 95.35 .+\mathrm{d}, 04.40 .-\mathrm{b}$
\end{abstract}

\section{INTRODUCTION}

The numerous attempts to give a theoretical description of the current accelerated expansion of the Universe are usually reduced to introducing an exotic form of matter called dark energy, which is believed to be responsible for such an acceleration. Whereas the true nature of dark energy is still unknown, different possibilities are being suggested to describe it. Perhaps one of the most promising approaches to addressing the origin of dark energy is a consideration of theories that include various fundamental fields [1, 2]. Possibilities are also being considered, including some that involve modified (non-Einstein) gravity theories [3, 4] or models with extra space dimensions [ [5, $\underline{6}]$. In any case, a description of the present acceleration of the Universe within the framework of the aforementioned approaches implies that one needs to choose the parameters entering these theories in such a way as to satisfy the current astronomical observations and laboratory experiments.

One of such approaches is the so-called chameleon paradigm, the essence of which, briefly, is as follows [7, 8]: Based on the assumption that scalar fields with a very small mass of order $H_{0} \sim 10^{-33} \mathrm{eV}$ do exist in the Universe, and they drive the current accelerated expansion, it is assumed that such fields may interact directly with ordinary matter and dark matter with gravitational strength. This leads to the fact that the scalar field acquires a mass which depends on the local background matter density. For example, on Earth, where the density is high, the mass of the field becomes $10^{30}$ times greater than the mass of this field on the cosmological background.

An important ingredient of the chameleon mechanism is the presence of two functions of the scalar field $\varphi$ : the potential energy $V(\varphi)$ and the function $f(\varphi)$ describing the direct coupling between the matter and the scalar field. These two functions are arbitrary, and they are chosen in such a way as to satisfy various laboratory and cosmological tests $7-10]$.

It is obvious that this arbitrariness in the choice of the mentioned field functions is due to lack of knowledge of the true nature of scalar fields filling the Universe (of course, assuming they do really exist in the present Universe and are responsible for its accelerated expansion). Perhaps the only thing one can do at this point is to try to find the restrictions imposed upon the form of these functions and values of the parameters appearing in them. Some work has already been done along these lines, particularly in the papers [11 -13], where the authors choose a generalized expression for the nonminimal coupling between the scalar field and the matter having the form $f(\varphi) L_{m}$, with $L_{m}$ being the Lagrangian of ordinary matter. In considering the evolution of the Universe they have used two different approaches: in Ref. [1] the functions $V(\varphi)$ and $f(\varphi)$ are taken to be arbitrary, and their parameters are chosen in such a way as to satisfy the current observational data. Another approach has been applied in [12, 13], where the authors have initially selected some particular form of cosmological evolution and found the functions $V(\varphi)$ and $f(\varphi)$ corresponding to such evolution.

On the other hand, the question may be asked: if the mechanism of direct coupling between a chameleon scalar field and ordinary matter is universal, i.e., it can be applied to any form of matter (be it ordinary matter or dark

\footnotetext{
*Email: vfolomeev@mail.ru
} 
matter), how will this affect the properties of various small-scale objects such as, for instance, usual stars? In Einstein gravity, compact configurations consisting of ordinary matter and scalar fields have been repeatedly considered earlier in the literature. The scalar fields may be self-interacting or not, and one can involve a fluid which interacts with the scalar field either only gravitationally [14, 15] or through direct coupling as well [16, 17]. In particular, in the recent papers [18 20] the changes in the structure of compact objects (polytropic stars) under the influence of a chameleon field have been investigated. It was shown that, depending on the properties of arbitrarily chosen functions $V(\varphi)$ and $f(\varphi)$, the properties of these configurations, such as total mass, distribution of matter, and size, depend strongly on the surrounding scalar field.

The purpose of the present paper is to demonstrate the possibility of determining the nonminimal coupling function $f(\varphi)$ which satisfies the current astronomical observations. Subsequently, this function will be used in modeling compact objects in order to clarify the influence of the presence of the chameleon scalar field on their physical characteristics. As an example, a polytropic star embedded in the chameleon scalar field will be considered.

The paper is organized as follows: In Sec. II the nonminimal coupling function $f(\varphi)$ is obtained, in general form, from cosmological considerations. In the particular case of the $\Lambda \mathrm{CDM}$ model this function is derived in Sec. IIC and, in Sec. III is applied to the estimation of the influence of the nonminimal coupling upon the properties of usual stars whose matter can interact directly with a chameleon scalar field. In the Appendix, we consider the effects related to the nonminimal coupling in the sense of their possible manifestation in laboratory experiments. Finally, in Sec. IV] we summarize and discuss the results obtained.

\section{II. $f$ FROM COSMOLOGY}

As pointed out in the Introduction, here the choice of the nonminimal coupling function $f(\varphi)$ will be done based on a consideration of the cosmological evolution of the Universe within the chameleon paradigm suggested in Ref. [11]. To do this, we choose the Lagrangian of the system in the form

$$
L=-\frac{c^{4}}{16 \pi G} R+\frac{\Delta}{2} \partial_{\mu} \varphi \partial^{\mu} \varphi-V(\varphi)+f(\varphi) L_{m} .
$$

Here $\varphi$ is the real scalar field with the potential $V(\varphi) ; \Delta= \pm 1$ corresponds to the usual or phantom scalar field, respectively; $L_{m}$ is the Lagrangian of ordinary matter (a perfect fluid). The case $f=1$ corresponds to no direct coupling between the fluid and scalar field, but even in this case the two sources are still coupled via gravity.

\section{A. About the Lagrangian $L_{m}$}

The Lagrangian $L_{m}$ can be chosen in a variety of ways. For example, in Ref. 21] the choice $L_{m}=-2 \varepsilon$ is used, and in Refs. [22, 23], $L_{m}=p$ ( $\varepsilon$ and $p$ are the energy density and the pressure of a fluid, respectively). By varying both these matter Lagrangians with respect to a metric, one obtains the same energy-momentum tensor of the perfect fluid in the conventional form. However, it can be shown that, for instance, for static configurations, these Lagrangians, being substituted in the general Lagrangian (11), will give different equations for an equilibrium configuration (the Tolman-Oppenheimer-Volkoff (TOV) equations). In the case of $L_{m}=p$, the TOV equation will have the same form as when $f=1$. On the other hand, the use of $L_{m}=-2 \varepsilon$ leads to the appearance of an extra term on the right-hand side of the TOV equation associated with the nonminimal coupling.

Of course, in the limit $f \rightarrow 1$, for both choices of $L_{m}$, the gravitating system of a scalar field plus ordinary matter reduces to the same uncoupled system in which the ordinary matter and the scalar field interact only gravitationally. But in the general case of $f=f(\varphi)$, these two systems will not be equivalent, giving equilibrium configurations with different properties. Apparently, at the present time, it is difficult to make a motivated choice amongst these Lagrangians $L_{m}$, or any other Lagrangians used in the literature (for the other possible Lagrangians, see, e.g., Ref. 24]). For this reason, a description of various systems with nonminimal coupling of the type $f(\varphi) L_{m}$ is made, in effect, using an ad hoc choice of $L_{m}$.

\section{B. Deriving $f$ in general form}

In this paper we choose $L_{m}=p$ which has been used in Refs. [18 20] in modeling astrophysical objects. Here this choice of $L_{m}$ will be used in studying the cosmological evolution of the present Universe. 
Consider the flat model with the metric

$$
d s^{2}=c^{2} d t^{2}-a^{2}(t) d l^{2},
$$

where $a(t)$ is the scale factor. Using the general Lagrangian (1), the corresponding energy-momentum tensor is (details are given in the appendix of [18])

$$
T_{i}^{k}=f\left[(\varepsilon+p) u_{i} u^{k}-\delta_{i}^{k} p\right]+\Delta \partial_{i} \varphi \partial^{k} \varphi-\delta_{i}^{k}\left[\frac{\Delta}{2} \partial_{\mu} \varphi \partial^{\mu} \varphi-V(\varphi)\right]
$$

where $\varepsilon$ and $p$ are the energy density and the pressure of the fluid, and $u^{i}$ is the 4 -velocity. Using the metric (2) and the energy-momentum tensor (3), the $\left(\begin{array}{l}0 \\ 0\end{array}\right)$ component of the Einstein equations is then given by

$$
\left(\frac{\dot{a}}{a}\right)^{2}=\frac{8 \pi G}{3 c^{2}}\left[f \varepsilon+\frac{\Delta}{2 c^{2}} \dot{\varphi}^{2}+V(\varphi)\right] .
$$

Here the dot denotes differentiation with respect to the cosmological time $t$. The equation for the scalar field $\varphi$ follows from the Lagrangian (1)

$$
\frac{1}{\sqrt{-g}} \frac{\partial}{\partial x^{i}}\left[\sqrt{-g} g^{i k} \frac{\partial \varphi}{\partial x^{k}}\right]=\Delta\left(-\frac{d V}{d \varphi}+L_{m} \frac{d f}{d \varphi}\right)
$$

and gives in the metric (2)

$$
\ddot{\varphi}+3 \frac{\dot{a}}{a} \dot{\varphi}=\Delta c^{2}\left(-\frac{d V}{d \varphi}+p \frac{d f}{d \varphi}\right) .
$$

One more equation can be obtained from the law of conservation of energy and momentum, $T_{i ; k}^{k}=0$. Taking the $i=0$ component of this equation gives

$$
\frac{d(f \varepsilon)}{d t}+3 \frac{\dot{a}}{a} f(\varepsilon+p)+p \frac{d f}{d t}=0 .
$$

Keeping in mind that $\varepsilon$ and $p$ are related by some equation of state, there are three unknown functions: $a, \varphi, \varepsilon$, and three equations (4), (6), and (7) for them.

Now we need to choose an equation of state for the matter content of the Universe. At the present time, it is believed that the main part of matter in the Universe (except dark energy) is in the form of dark matter. The most popular hypothesis in modeling dark matter is the assumption that its equation of state corresponds to cold (dust) matter [1]. Such matter is either pressureless or has a very small pressure (see, e.g., Ref. [25]). In hydrodynamical language, in cosmology, the relation between the pressure and energy density is usually taken in the simplest form as $p=w \varepsilon$, where $w=$ const. Assuming $w \approx 0$ at the present time, a description of the evolution of the Universe is carried out.

Here we initially start with the assumption that the pressure of the matter filling the Universe is small compared with its energy density. To apply the results obtained from cosmology for modeling compact astrophysical objects, let us choose a polytropic equation of state which is widely used in astrophysics. This equation of state may be taken in the following parametric form:

$$
p=K \rho^{1+1 / n}, \quad \varepsilon=\rho c^{2} .
$$

Here the constant $n$ is called the polytropic index and is related to the specific heat ratio $\gamma$ via $n=1 /(\gamma-1), \rho$ is the rest-mass density, and $K$ is an arbitrary constant whose value depends on the properties of the fluid under consideration. Substituting this equation of state in (7), its general solution will be

$$
\frac{\rho}{\left(1+\alpha \rho^{1 / n}\right)^{n}}=\frac{\rho_{0}}{f a^{3}}, \quad \alpha=K / c^{2},
$$

where $\rho_{0}$ is an integration constant. The smallness of the pressure implies that the term in the denominator $\alpha \rho^{1 / n} \ll 1$. Then, neglecting this term, we obtain from Eq. (9) the following approximate expression for the mass density:

$$
\rho \approx \frac{\rho_{0}}{f a^{3}} .
$$


In the limit $f \rightarrow 1$, i.e., in the absence of the nonminimal coupling, this expression reduces to the usual dependence for the density of dust matter as a function of the scale factor.

For the subsequent calculations, it is convenient to introduce new dimensionless variables

$$
\xi=B t, \quad B=\sqrt{8 \pi G \rho_{0}}, \quad \phi(\xi)=\left(\frac{8 \pi G}{c^{4}}\right)^{1 / 2} \varphi(t),
$$

where $B$ has the dimensions of inverse time. Using these variables and taking into account the equation of state (8) and the expression (10), the system of Eqs. (41) and (6) takes the form

$$
\begin{aligned}
& 3\left(\frac{a^{\prime}}{a}\right)^{2}=\frac{1}{a^{3}}+\frac{\Delta}{2} \phi^{\prime 2}+\tilde{V}, \\
& \phi^{\prime \prime}+3 \frac{a^{\prime}}{a} \phi^{\prime}=\Delta\left(-\frac{d \tilde{V}}{d \phi}+\frac{\sigma}{f^{\gamma} a^{3 \gamma}} \frac{d f}{d \phi}\right) .
\end{aligned}
$$

Here the prime denotes differentiation with respect to the dimensionless time $\xi ; \gamma=1+1 / n, \tilde{V}=V /\left(\rho_{0} c^{2}\right), \sigma=$ $p_{0} /\left(\rho_{0} c^{2}\right)=K \rho_{0}^{1 / n} / c^{2}$, where $\rho_{0}, p_{0}$ refer to the current values of the mass density and the pressure, respectively. Note that in our low-pressure approximation the parameter $\sigma \ll 1$. Also, in this approximation, the first term on the right-hand side of Eq. (12) always plays the role of dustlike matter, independently of the form of the function $f$. In turn, the latter, together with $V$, will describe an evolution of all other forms of matter, including dark energy.

Next, using Eqs. (12) and (13), let us find the form of the function $f$ expressed in terms of the scale factor $a$. To do this, rewrite Eq. (13) as

$$
\frac{1}{2} \frac{d\left(\phi^{\prime 2}\right)}{d \xi}+3 \frac{a^{\prime}}{a} \phi^{\prime 2}=\Delta\left(-\frac{d \tilde{V}}{d \xi}+\frac{\sigma}{f^{\gamma} a^{3 \gamma}} \frac{d f}{d \xi}\right)
$$

Expressing from (12)

$$
\phi^{\prime 2}=2 \Delta\left[3\left(\frac{a^{\prime}}{a}\right)^{2}-\frac{1}{a^{3}}-\tilde{V}\right]
$$

and substituting this in (14), one can find the following dependence of $f$ on $a$ :

$$
f^{1-\gamma}=6 \frac{1-\gamma}{\sigma} \int a^{\prime} a^{3 \gamma-4}\left(a^{2} a^{\prime \prime}+2 a a^{\prime 2}-\frac{1}{2}-a^{3} \tilde{V}\right) d \xi
$$

Then, if $a$ and $\tilde{V}$ are known as functions of the time $\xi$, it is possible to find the corresponding dependence $f=f(\xi)$.

\section{Expression for $f$ in the case of the $\Lambda$ CDM model}

We now will demonstrate how to obtain an expression for $f$ in the particular case of $\tilde{V}=0$ (a massless scalar field), and when the ansatz for the scale factor $a$ is chosen in the form of the well-known $\Lambda$ CDM model [1]

$$
a(\xi)=a_{0}\left(\frac{\Omega_{m}}{\Omega_{\Lambda}}\right)^{1 / 3}\left(\sinh \frac{\sqrt{3}}{2} \sqrt{\frac{\Omega_{\Lambda}}{\Omega_{m}}} \xi\right)^{2 / 3}
$$

Here $\Omega_{m}=8 \pi G \rho_{0} / 3 H_{0}^{2}, \Omega_{\Lambda}=c^{2} \Lambda / 3 H_{0}^{2}$, where $H_{0}=H\left(t=t_{0}\right)$ and $a_{0}=a\left(t=t_{0}\right)$ are the present values of the Hubble parameter and the scale factor, respectively. This expression for $a$ smoothly interpolates between a matter-dominated Universe in the past $\left(a \propto t^{2 / 3}\right)$ and accelerated expansion in the future $(a \propto \exp \sqrt{\Lambda / 3} c t)$.

Setting $a_{0}=1$ at the present epoch and substituting (17) in (16), one can find

$$
f^{1-\gamma}=2 \frac{1-\gamma}{\sigma \gamma}\left(\frac{\Omega_{\Lambda}}{\Omega_{m}}\right)^{1-\gamma}\left(\sinh \frac{\sqrt{3}}{2} \sqrt{\frac{\Omega_{\Lambda}}{\Omega_{m}}} \xi\right)^{2 \gamma}
$$


In turn, we have from (15)

$$
\phi^{\prime 2}=2 \Delta \frac{\Omega_{\Lambda}}{\Omega_{m}} \Rightarrow \phi=\sqrt{2 \frac{\Omega_{\Lambda}}{\Omega_{m}}} \xi \quad \text { and } \quad \xi=\frac{1}{\sqrt{2}} \sqrt{\frac{\Omega_{m}}{\Omega_{\Lambda}}} \phi .
$$

(Here we have set $\Delta=+1$ in order to ensure that the functions $f$ and $a$ obtained below will be real.) Note here that by substituting the above derivative of the scalar field into Eq. (12) and multiplying it by $\Omega_{m}$, we get the known equation for the $\Lambda$ CDM model. That is, in the case under consideration, the gradient of the scalar field plays the role of the $\Lambda$-term.

Substituting the expression for $\xi$ from (19) in Eqs. (17) and (18), we find the final formulas for $f$ and $a$ as functions of the scalar field $\phi$

$$
\begin{aligned}
& a=\left(\frac{\Omega_{m}}{\Omega_{\Lambda}}\right)^{1 / 3}\left(\sinh \sqrt{\frac{3}{8}} \phi\right)^{2 / 3} \\
& f=\frac{\Omega_{\Lambda}}{\Omega_{m}}\left[-\frac{\sigma(n+1)}{2}\right]^{n}\left(\sinh \sqrt{\frac{3}{8}} \phi\right)^{-2(n+1)}
\end{aligned}
$$

Using (20), one can find the current value of the scalar field, $\phi_{0} \equiv \phi\left(t=t_{0}\right)$. To do this, setting $a=a_{0}=1$ and choosing, for definiteness, $\Omega_{\Lambda}=0.7$ and $\Omega_{m}=0.3$, from the solution of the transcendental equation (20), we have

$$
\phi_{0} \approx 1.975, \quad \xi_{0} \approx 0.915
$$

This $\xi_{0}$ corresponds to $t_{0} \approx 12.95$ Gyrs.

\section{CHAMELEON STAR}

We now will proceed to a consideration of a compact configuration (a star) consisting of ordinary (polytropic) matter with the equation of state (8) and of a scalar field nonminimally interacting with this matter. In Ref. [18] this type of gravitating configuration of a polytropic fluid nonminimally coupled to a scalar field was called a "chameleon star."

As a nonminimal coupling function, we will use the expression (21) obtained in the preceding section. We proceed from the assumption that the cosmological chameleon scalar field penetrates into the star and interacts with the star's matter through the same universal coupling function $f$ from (21). That is, we assume that the matter of the star, which is embedded in the external, cosmological, homogeneous scalar field, feels its presence not only gravitationally, but also through the nonminimal coupling. Then, the structure of the star is also defined by the distribution of the scalar field, and correspondingly by $f$, inside the star.

\section{A. General equations for hydrostatic equilibrium configurations}

We choose the static spherically symmetric metric in the form

$$
d s^{2}=e^{\nu} c^{2} d t^{2}-e^{\lambda} d r^{2}-r^{2} d \Omega^{2}
$$

where $\nu$ and $\lambda$ are functions of the radial coordinate $r$, and $d \Omega^{2}$ is the metric on the unit two-sphere. The $\left(\begin{array}{l}0 \\ 0\end{array}\right)$ and $\left(\begin{array}{l}1 \\ 1\end{array}\right)$ components of the Einstein equations for the metric (23) and the energy-momentum tensor (3) are then given by

$$
\begin{aligned}
& G_{0}^{0}=-e^{-\lambda}\left(\frac{1}{r^{2}}-\frac{\lambda^{\prime}}{r}\right)+\frac{1}{r^{2}}=\frac{8 \pi G}{c^{4}}\left(f \varepsilon+\frac{1}{2} e^{-\lambda} \varphi^{\prime 2}+V\right) \\
& G_{1}^{1}=-e^{-\lambda}\left(\frac{1}{r^{2}}+\frac{\nu^{\prime}}{r}\right)+\frac{1}{r^{2}}=\frac{8 \pi G}{c^{4}}\left(-f p-\frac{1}{2} e^{-\lambda} \varphi^{\prime 2}+V\right),
\end{aligned}
$$

where the prime denotes differentiation with respect to $r$. (As in the previous section, we have again set $\Delta=+1$.) Here the energy density $\varepsilon$ and the pressure $p$ refer now to the matter of the star (but not to the cosmological matter), but the scalar field, in the spirit of the chameleon paradigm, remains the same as in cosmology. Our purpose will be 
to clarify the influence that this cosmological scalar field may have on the inner structure and characteristics of such compact objects as usual stars.

Equations (24) and (25) must be supplemented by an equation for the scalar field, which follows from (5) and has the following form in the metric (23):

$$
\varphi^{\prime \prime}+\left[\frac{2}{r}+\frac{1}{2}\left(\nu^{\prime}-\lambda^{\prime}\right)\right] \varphi^{\prime}=e^{\lambda}\left(\frac{d V}{d \varphi}-p \frac{d f}{d \varphi}\right) .
$$

One more equation follows from the law of conservation of energy and momentum, $T_{i ; k}^{k}=0$. Taking the $i=1$ component of this equation gives

$$
\frac{d p}{d r}=-\frac{1}{2}(\varepsilon+p) \frac{d \nu}{d r}
$$

To perform the subsequent calculations, it is convenient to rewrite the equation of state (8) in terms of the new variable $\theta$ related to the density $\rho$ at the given point and the central density $\rho_{c}$ by (see, e.g., Ref. [26])

$$
\rho=\rho_{c} \theta^{n_{s}} \Rightarrow p=K \rho^{\gamma_{s}}=K \rho^{1+1 / n_{s}}=K \rho_{c}^{1+1 / n_{s}} \theta^{n_{s}+1} .
$$

Notice that $\theta$ must be "normalized" to unity when $r=0$. Using (28) in Eq. (27) leads to

$$
2 \sigma_{s}\left(n_{s}+1\right) \frac{d \theta}{d r}=-\left(1+\sigma_{s} \theta\right) \frac{d \nu}{d r}
$$

with $\sigma_{s}=K \rho_{c}^{1 / n_{s}} / c^{2}=p_{c} /\left(\rho_{c} c^{2}\right)$ and $p_{c}$ is the pressure of the fluid at the center of the configuration. The index $s$ ("star") on $\sigma, n$, and $\gamma$ is introduced here to distinguish these parameters from those used in Sec. II in describing the matter content of the Universe. In both cases, the relativistic parameters $\sigma$ and $\sigma_{s}$ characterize the ratio of the pressure at a given point (in the case of a star, at the center of the configuration), or at a given instant of time (in the case of cosmology, at the present time) to the energy density. Since the expression for the coupling function $f$ from (21) has been obtained in the low-pressure approximation, i.e., is valid only when $\sigma \ll 1$, therefore, it is natural that in the case of a star this expression is also applicable only when $\sigma_{s} \ll 1$.

Equation (29) may be integrated to give $e^{\nu}$ in terms of $\theta$

$$
e^{\nu}=e^{\nu_{c}}\left(\frac{1+\sigma_{s}}{1+\sigma_{s} \theta}\right)^{2\left(n_{s}+1\right)}
$$

where $e^{\nu_{c}}$ is the value of $e^{\nu}$ at the center of the configuration where $\theta_{c}=1$. The integration constant $\nu_{c}$ corresponds to the value of $\nu$ at the center of the configuration. It is determined by requiring $e^{\nu}=1$ at infinity, i.e., that the space-time be asymptotically flat.

We introduce a new function $M(r)$

$$
e^{-\lambda}=1-\frac{2 G M(r)}{c^{2} r} .
$$

$M(r)$ is the mass of the configuration in the range $0 \leq r \leq r_{1}$, where $r_{1}$ is the boundary of the fluid where $\theta=0$. Also, we introduce dimensionless variables

$$
x=A r, \quad v(x)=\frac{A^{3} M(r)}{4 \pi \rho_{c}}, \quad \phi(x)=\left[\frac{4 \pi G}{\sigma_{s}\left(n_{s}+1\right) c^{4}}\right]^{1 / 2} \varphi(r), \quad \text { where } \quad A=\left[\frac{4 \pi G \rho_{c}}{\sigma_{s}\left(n_{s}+1\right) c^{2}}\right]^{1 / 2},
$$

where $A$ has the dimensions of inverse length. With this, we can rewrite Eqs. (24) and (25) in the form

$$
\begin{aligned}
\frac{d v}{d x} & =x^{2}\left\{f \theta^{n_{s}}+\frac{1}{2}\left[1-2 \sigma_{s}\left(n_{s}+1\right) \frac{v}{x}\right]\left(\frac{d \phi}{d x}\right)^{2}+\tilde{V}\right\}, \\
x^{2} \frac{1-2 \sigma_{s}\left(n_{s}+1\right) v / x}{1+\sigma_{s} \theta} \frac{d \theta}{d x} & =x^{3}\left[f \theta^{n_{s}}\left(1-\sigma_{s} \theta\right)+2 \tilde{V}-\frac{1}{x^{2}} \frac{d v}{d x}\right]-v,
\end{aligned}
$$

where $\tilde{V}=V /\left(\rho_{c} c^{2}\right)$ is the dimensionless potential energy of the field. Next, using (29), one can rewrite Eq. (26) as follows:

$$
\begin{aligned}
\frac{d^{2} \phi}{d x^{2}}+ & \left\{\frac{2}{x}-\frac{\sigma_{s}\left(n_{s}+1\right)}{1+\sigma_{s} \theta}\left[\frac{d \theta}{d x}+\frac{1+\sigma_{s} \theta}{1-2 \sigma_{s}\left(n_{s}+1\right) v / x} \frac{1}{x}\left(\frac{d v}{d x}-\frac{v}{x}\right)\right]\right\} \frac{d \phi}{d x} \\
& =\left[1-2 \sigma_{s}\left(n_{s}+1\right) \frac{v}{x}\right]^{-1}\left(\frac{d \tilde{V}}{d \phi}-\sigma_{s} \theta^{n_{s}+1} \frac{d f}{d \phi}\right) .
\end{aligned}
$$


Thus, the static configuration under consideration is described by the three equations (33)-(35).

\section{B. The particular choice of $f$ in the form (21)}

We now apply Eqs. [33)-(35) for describing the inner structure of our configuration. In Ref. [18], this system was used in studying similar configurations (chameleon stars) when the coupling function $f$ was chosen arbitrarily. It was shown that the presence of the nonminimal interaction leads to substantial changes both in the radial matter distribution of the stars and in the star's total mass.

To demonstrate the influence of the nonminimal coupling derived in Sec. П on the properties of a compact configuration, here we will consider a case when the potential energy is absent, i.e., $V(\phi)=0$. As mentioned above, we assume that the scalar field, being initially homogeneous (i.e., cosmological), in the presence of star's matter, begins to interact with the matter both gravitationally and through the nonminimal coupling. Our purpose will be to study the possible effects of this interaction.

Note that, in the absence of the nonminimal coupling, the choice $V(\phi)=0$ corresponds to the case of a massless scalar field. However, one can see from the right-hand side of Eq. (35) that the presence of the nonminimal coupling leads to the presence of an additional term of the form $\sigma_{s} \theta^{n_{s}+1} d f / d \phi$.

Choosing $f$ in the form (21), Eqs. (33)-(35) are to be solved for given $\sigma_{s}, n_{s}$, and also cosmological parameters $\sigma$, $n, \Omega_{\Lambda}$, and $\Omega_{m}$ subject to the boundary conditions in the vicinity of the center of the configuration $x=0$,

$$
\theta \approx \theta_{c}+\frac{\theta_{2}}{2} x^{2}, \quad v \approx v_{3} x^{3}, \quad \phi \approx \phi_{c}+\frac{\phi_{2}}{2} x^{2},
$$

where $\phi_{c}$ corresponds to the central value of the scalar field $\phi, \theta_{c}$ is normalized to be unity at the center, $\theta_{c} \equiv \theta(x=$ $0)=1$. The values of the coefficients $\phi_{2}, v_{3}$, and $\theta_{2}$ are defined from Eqs. (35), (33), and (34), respectively, as

$$
\phi_{2}=-\frac{1}{3} \sigma_{s}\left(\frac{d f}{d \phi}\right)_{c}, \quad v_{3}=f_{c} / 3, \quad \theta_{2} \approx-\frac{f_{c}}{3}\left(1+4 \sigma_{s}\right),
$$

where the index $c$ corresponds to central values.

Since the cosmological scalar field, $\phi(\xi)$, and that used in modeling a star, $\phi(x)$, are measured in different units, then, to find the relation between them, we compare their definitions given by expressions (11) and (32), respectively. Thus we have

$$
\phi(\xi)=\sqrt{2 \sigma_{s}\left(n_{s}+1\right)} \phi(x) .
$$

Substituting this expression in (21), we finally obtain

$$
f=\frac{\Omega_{\Lambda}}{\Omega_{m}}\left[-\frac{\sigma(n+1)}{2}\right]^{n}\left(\sinh \sqrt{\frac{3}{4} \sigma_{s}\left(n_{s}+1\right)} \phi(x)\right)^{-2(n+1)} .
$$

As a result, the static configuration under consideration is described by the system of Eqs. (33)-(35) together with the boundary conditions (36) and the function $f$ from (38). These equations, however, cannot be integrated analytically, and have to be solved numerically. The solutions were started near the vicinity of the center of the configuration (i.e., near $x \approx 0$ ) and solved out to a point $x=x_{1}$, where the function $\theta$ became zero. Since our spherical fluid configuration was taken to be embedded in an external, homogeneously distributed scalar field, we also needed to require that at the boundary, $x=x_{1}$, the value of the varying scalar field from the inside of the star, $\phi(x)$, matched the exterior value $\phi_{\text {ext }}$. In this case, of course, the gradient of the scalar field, generally speaking, although small, is not strictly equal to zero. It requires continuing the solutions outside the fluid. Formally, the gradient goes to zero only asymptotically, i.e., as $x \rightarrow \infty$. However, the numerical calculations indicate that for small values of the parameters $\sigma$ and $\sigma_{s}$ used in this paper, the gradient of the scalar field is virtually equal to zero along the radius of the configuration. Then the form of the external scalar field is determined by matching it to the interior solution at the surface of the star. The junction conditions, which follow from the requirement that the field equations be satisfied on the boundary of the star, require that $\phi(x)$ be continuous. In our case of small scalar-field gradients, this can be done with sufficient accuracy by equating the interior and exterior values of the scalar field at $x=x_{1}$, i.e., by requiring that $\phi\left(x_{1}\right) \approx \phi_{\text {ext }}$.

In turn, for a complete description of the system under consideration, it is also necessary to solve external Einstein's equations for the metric functions $\nu$ and $\lambda$ from (23). This can be easily done in the manner described in Ref. [20]. 


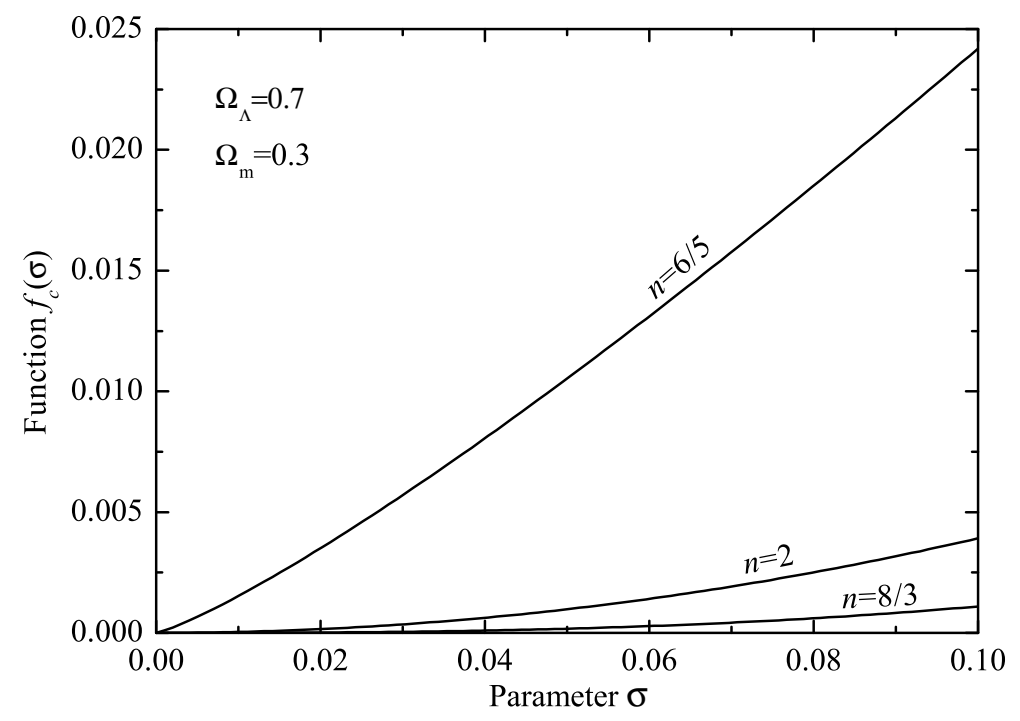

FIG. 1: The function $f_{c}$ given by Eq. (42) versus $\sigma$ for $n=6 / 5,2,8 / 3$.

However, for the problems being considered in this paper, knowledge of the behavior of the exterior solutions is not necessarily needed. That is why, in order not to encumber the paper, we do not show this procedure here.

Thus, the procedure for finding solutions is as follows: for given values of $\sigma, \sigma_{s}, \Omega_{\Lambda}, \Omega_{m}, n$, and $n_{s}$, we choose the central value of the scalar field, $\phi_{c}$, such that $\theta$ goes to zero and $\phi\left(x_{1}\right) \approx \phi_{\text {ext }}$ at some finite value of $x=x_{1}$. The fact that the configuration is embedded in the external cosmological scalar field $\phi_{0}$ implies that $\phi_{\text {ext }}$ must be equal to $\phi_{0} / \sqrt{2 \sigma_{s}\left(n_{s}+1\right)}$, where $\phi_{0}$ is taken from (22) [the coefficient $\sqrt{2 \sigma_{s}\left(n_{s}+1\right)}$ appears because of the relation (37)] ]. This allows one to determine definitely the value of $\phi_{c}$ for such configurations.

One can see from the expression (38) that the function $f$ can be positive, negative, or imaginary depending on the value of $n$. Physically reasonable values of $f$ are either positive or negative. However, the numerical calculations show that the parameters used in the paper give finite size solutions only for positive values of this function. To provide the positivity of $f$, it is necessary to take $n$ either even or equal to

$$
n=\frac{2 l}{2 m+1},
$$

where $m$ and $l$ are integers. Proceeding from this choice, we get the results described below.

In order not to encumber the paper, in further calculations the polytropic indexes $n$ and $n_{s}$ are taken to be equal to each other. The calculations indicate that even if this is not the case, the qualitative behavior of the solutions remains the same. Keeping this in mind, we present here the results of numerical calculations with the following values of the parameters $n$ and $n_{s}: n=n_{s}=6 / 5 ; 2 ; 8 / 3$ (for convenience, we hereafter drop the index $s$ on $n_{s}$ ). These values cover the range $1.2<n \lesssim 2.67$, which is often used in modeling usual stars. The parameters $\Omega_{\Lambda}$ and $\Omega_{m}$ are chosen to be $\Omega_{\Lambda}=0.7, \Omega_{m}=0.3$, as before. In the approximation used here, the relativistic parameters $\sigma, \sigma_{s}$ are taken to be much less than unity: $\sigma \lesssim 0.1, \sigma_{s} \lesssim 0.05$.

With this choice of the parameters, the numerical calculations indicate that the scalar field $\phi(x)$ and the coupling function $f$ are virtually constant along the radius of the configuration under consideration. This allows us to rewrite the system (33)-(35) with $f$ from (38), using the approximation $\phi^{\prime} \approx 0$ and $f(x) \approx f_{c}=$ const, in the following form:

$$
\begin{aligned}
\frac{d v}{d x} & =f_{c} x^{2} \theta^{n} \\
x^{2} \frac{1-2 \sigma_{s}(n+1) v / x}{1+\sigma_{s} \theta} \frac{d \theta}{d x} & =x^{3}\left[f_{c} \theta^{n}\left(1-\sigma_{s} \theta\right)-\frac{1}{x^{2}} \frac{d v}{d x}\right]-v .
\end{aligned}
$$

Then, introducing the new dimensionless radial coordinate $x=\tilde{x} / \sqrt{f_{c}}$ and mass function $v=\tilde{v} / \sqrt{f_{c}}$, we are able to get rid of $f_{c}$ in these equations. Thus, mathematically this system of equations becomes equivalent to that of describing usual polytropic stars with an equation of state in the form (8) and in the absence of a scalar field. Such configurations 
were investigated in detail in the work of Tooper [27] who found regular, relativistic starlike configurations. These starlike solutions of [27] were found for the values of the parameters $0 \leq \sigma_{s} \leq 0.75$ and $1 \leq n \leq 3$.

In our case we are dealing with approximately the same range of $n$, but with small values of $\sigma_{s}$. Correspondingly, the size and the mass of our configurations are $1 / \sqrt{f_{c}}$ times the values of those considered in [27] in the absence of a scalar field. That is, in our approximation the value of $f_{c}$ is the primary determining parameter of the problem.

It is obvious that $f_{c}$ must depend on the values of three parameters: $n, \sigma$, and $\sigma_{s}$. However, the numerical calculations show that, for fixed $n$ and varying $\sigma$ and $\sigma_{s}$, the argument of the hyperbolic sine in (38) is a slowly varying function of $\sigma_{s}$. That is why, to a good approximation, this argument may be regarded as a constant, that allows to rewrite the function $f_{c}(\sigma)$ in the following approximate form:

$$
f_{c}(\sigma) \approx \bar{f}_{n}\left[-\frac{\sigma(n+1)}{2}\right]^{n}, \quad \text { with } \quad \bar{f}_{n}=\frac{\Omega_{\Lambda}}{\Omega_{m}}\left(\sinh \sqrt{\frac{3}{4} \sigma_{s}(n+1)} \phi(x)\right)^{-2(n+1)} \approx \text { const },
$$

where $\bar{f}_{n}$ is approximately constant along the radius of the configuration for any fixed $n$. For the values of $n$ used here, the coefficient $\bar{f}_{n}$ has the following numerical values: $\bar{f}_{n=6 / 5} \approx 0.342, \bar{f}_{n=2} \approx 0.174$, and $\bar{f}_{n=8 / 3} \approx 0.101$. The numerical calculations indicate that, using these values, the approximation for $f_{c}(\sigma)$ in the form (42) is applicable with good accuracy when $\sigma \lesssim 0.1$ and $\sigma_{s} \lesssim 0.05$.

Figure 1 shows the function $f_{c}(\sigma)$ from (42). One can see from this figure that the value of $f_{c}(\sigma)$, being always much less than unity, grows with increasing $\sigma$ for all $n$. Since the size and the mass of the configurations under consideration are inversely proportional to the square root of $f_{c}(\sigma)$, then its small values for any $\sigma$ and $n$ correspond to large sizes and masses compared with configurations without a scalar field considered in Ref. [27]. The numerical results obtained in [27] are written in terms of dimensionless variables in the form (11) for arbitrary values of the equation-of-state parameters $K, n$, and the central density $\rho_{c}$. Correspondingly, the concrete values of physical characteristics (masses and sizes) can be obtained in dimensional form by giving the corresponding values of the mentioned parameters. But in any case, the size and the mass of configurations with a scalar field considered in the present paper increase catastrophically in the limit of very small $\sigma$, corresponding to dustlike cosmological matter.

\section{SUMMARY AND DISCUSSION}

It is now believed that various scalar fields may exist in the present Universe. Based on the assumption that they can interact directly (nonminimally) with ordinary matter and dark matter with gravitational strength, this paper studies the possible influence that such fields may have on the properties of compact gravitating configurations (stars) consisting of ordinary matter. The choice of the nonminimal coupling function $f$ is made from a consideration of the evolution of the Universe within the framework of chameleon cosmology. Then the total Lagrangian initially contains a term describing a nonminimal coupling between the scalar field $\varphi$ and the matter in the general form $f(\varphi) L_{m}$, with $L_{m}$ being the Lagrangian of ordinary matter [see Eq. (11)]. By choosing the arbitrary function $f(\varphi)$, one might try to describe the evolution of the present Universe.

In the present paper the choice of this function is based on two propositions: (i) The pressure of the matter filling the Universe is small compared with its energy density. (ii) The evolution of the cosmological scale factor $a$ is determined by the observational data.

To model the matter content of the Universe (namely, ordinary matter plus dark matter), we choose a polytropic equation of state in the form (8). This choice is caused by our wish to use the results obtained from cosmology in modeling compact objects (see below). For this equation of state, in the low-pressure approximation, it is possible to find a general expression for $f$ as a function of $a$ in the form (16). Next, using the well-known $\Lambda$ CDM ansatz for the scale factor, we find $f$ as a function of the scalar field in the final form (21).

Our next step is to consider the influence that the obtained nonminimal coupling may have on the properties of usual stars. While so doing, we start from the assumption that a star's matter is described by the same equation of state (8) and also can interact directly with a cosmological scalar field. One can consider such a configuration as embedded in the scalar field which penetrates into the star and interacts with its matter through the function $f$.

Depending on a concrete form of $f$, such a direct coupling results in more or less significant changes in the structure and properties of a star (see Refs. 18 20]). In our low-pressure approximation, when the pressures both of the cosmological matter and of the star's fluid are much less than their energy densities, the function $f$, as well as the scalar field, may be regarded as practically constant along the radius of the star: $f(x) \approx f_{c}=$ const. This allows one to rewrite the system of Eqs. (33)-(35), describing a static configuration, in the form which is mathematically identical to that without a scalar field [see Eqs. (40) and (41)]. This corresponds to the fact that the size and the mass of our configurations are $1 / \sqrt{f_{c}}$ times the values obtained in Ref. [27], where configurations with the same equation of state, but without a scalar field, have been considered. But since in our case the values of $f_{c}$ are always much 
less than unity, the size and the mass of configurations with the chameleon scalar field are always much greater than those of configurations without a scalar field. It is seen from Fig. 1 that this effect is especially strong for small values of the cosmological parameter $\sigma$ which correspond to the vanishing pressure of the matter filling the Universe (dark matter plus ordinary matter). This gives rise to the fact that the size and the mass of compact configurations with the chameleon scalar field increase catastrophically compared with configurations without a scalar field.

Does this unusual behavior of the characteristics of the compact configurations considered here inevitably imply that such chameleon cosmology is nonviable? The answer to this question might be given in the following way: As one can see from Fig. 1, $f_{c}$ increases with increasing $\sigma$, and correspondingly the size and the mass of our configurations decrease. One might expect that the subsequent growth of $\sigma$, when the low-pressure approximation is not already valid, and the function $f$ is not constant throughout the star, will provide the possibility of obtaining physically reasonable sizes and masses of configurations supported by the chameleon scalar field. At least the previous studies with an arbitrary choice of $f$ performed in Refs. [18 20] indicate that one can obtain sizes and masses comparable to those of usual stars without a scalar field. However, such a problem with $f$ derived from cosmological considerations must be studied separately beyond the framework of the low-pressure approximation used in the present paper.

Thus, within the framework of chameleon cosmology considered here, polytropic stars supported by the cosmological chameleon scalar field will have physically reasonable sizes and masses only if we suppose that the pressure of the matter filling the Universe is nonvanishing. However, in the case of relatively small pressures considered here, this does not lead to any significant changes from the point of view of conventional cosmology. Indeed, it is seen from the cosmological Einstein equation (12) that its right-hand side is identical in form to the source of matter in the form of dust plus the terms containing the scalar field which describe dark energy, regardless of its form. In the particular case of the $\Lambda \mathrm{CDM}$ ansatz for the scale factor considered here, the massless scalar field gave the contribution which is equivalent to the $\Lambda$-term.

In the case when pressures both of the matter content of the Universe and of star's matter are not small compared with their energy densities, one would expect that the situation will change. On the one hand, one can expect to get more reasonable sizes and masses of compact configurations. On the other hand, from the point of view of cosmology, matter sources of the Einstein equations would be expected generally to have a different form from that of usually used in modeling the evolution of the Universe. But these questions should be considered in further studies.

We have also addressed the issue of how the effects related to the nonminimal coupling could be manifested in laboratory experiments. One such effect is the occurrence of extra accelerations whose values must lie within the restrictions imposed by various experiments. Proceeding from a consideration of the equation of motion for a fluid element in a given gravitational field, in the Appendix the restriction imposed by the Eötvös-type experiments is derived [see Eq. A.5 ] ] Despite the fact that the nonminimal function $f$ obtained here leads to the unusual behavior of the characteristics of compact configurations, we use this function to demonstrate the theoretical possibility of how one can estimate extra accelerations which appear in the Earth's atmosphere because of the presence of the nonminimal coupling. It is shown that the condition A.5 is satisfied in principle [see Eq. (A.14)]. In future work, we plan to perform a consideration of these questions using such a nonminimal coupling function, which gives a more adequate description of the characteristics of compact configurations.

\section{Acknowledgements}

The author is grateful to the Research Group Linkage Programme of the Alexander von Humboldt Foundation for the support of this research. This work was partially supported by a grant in fundamental research in natural sciences by the Ministry of Education and Science of Kazakhstan.

\section{Appendix A: Effects of the nonminimal coupling and laboratory constraints}

In this appendix we address the question of how the effects related to the presence of the nonminimal coupling in the system described by the Lagrangian (1) could manifest themselves in laboratory experiments.

To illustrate this, consider the equation of motion for a fluid element in a gravitational field. We derive this equation using the energy-momentum tensor (3) which can be rewritten in the form

$$
T^{\mu \nu}=f T^{\mu \nu(\mathrm{f})}+T^{\mu \nu(\mathrm{sf})},
$$

where

$$
T^{\mu \nu(\mathrm{f})}=(\varepsilon+p) u^{\mu} u^{\nu}-g^{\mu \nu} p, \quad T^{\mu \nu(\mathrm{sf})}=\Delta \partial^{\mu} \varphi \partial^{\nu} \varphi-g^{\mu \nu}\left[\frac{\Delta}{2} \partial_{i} \varphi \partial^{i} \varphi-V(\varphi)\right]
$$


Here the total energy-momentum tensor is divided into two parts denoted by the indexes (f) and (sf) which refer to the fluid and the scalar field, respectively. On operating a covariant derivative on (A.1), we have

$$
T_{; \nu}^{\mu \nu}=f T_{; \nu}^{\mu \nu(\mathrm{f})}+T^{\mu \nu(\mathrm{f})} f_{; \nu}+T_{; \nu}^{\mu \nu(\mathrm{sf})} .
$$

By virtue of the scalar field equation (5), the third term on the right-hand side of this expression vanishes. Projecting two remaining terms onto the direction of the fluid 4-velocity $u_{\mu}$, one can obtain the following equation of motion for a fluid element:

$$
\frac{d^{2} x^{\mu}}{d s^{2}}+\Gamma_{\alpha \nu}^{\mu} \frac{d x^{\alpha}}{d s} \frac{d x^{\nu}}{d s}=-\frac{p_{; \nu}+p(\ln f)_{; \nu}}{\varepsilon+p}\left(u^{\mu} u^{\nu}-g^{\mu \nu}\right) .
$$

The first term in the numerator on the right-hand side, $p_{; \nu}$, is the pressure gradient that also appears in usual non-chameleon models and describes the force exerted on a fluid element due to the fluid pressure (it is not to be attributed to the coupling between the matter and the scalar field, nor does it signal any new effect). The presence of this term forces particles of matter, within which there are pressures that generally move along lines that are not geodesics. The second term in the numerator, $p(\ln f)_{;}$, is provided by the presence of the nonminimal coupling in the system. One can see that its influence on the motion of a test particle is determined both by the magnitude of the fluid pressure and the gradient of the nonminimal coupling function $f$. In the general case when magnitudes of the pressure and the gradient of $f$ are not small, one might expect that substantial extra accelerations in the system described by the Lagrangian (1) will appear.

The presence of such extra accelerations implies that the theory may exhibit violations of the weak equivalence principle. The most stringent constraints on possible violations of the equivalence principle derive from experiments performed on Earth. These experiments test the universality of free fall, the prediction that all bodies in a uniform gravitational field have exactly the same gravitational acceleration. The universality of free fall has been tested to roughly 1 part in $10^{12}$ [26] using laboratory test bodies in the gravitational fields of the Earth and the Sun. Another sort of experiments is connected with precise measurements by using lunar laser-ranging to compare the accelerations of the Earth and Moon toward the Sun. These experiments constraint the difference in free-fall acceleration to be less than approximately one part in $10^{13}$ (see, e.g., Ref. [28]).

Then, proceeding from the requirement to satisfy the aforementioned laboratory experiments, one must make sure that the effects related to the presence of the nonminimal coupling do not lead to a contradiction with the experiments. To check this, we will estimate below the value of extra acceleration which appears in the presence of the nonminimal coupling with the function $f$ given by the expression (21). But before doing that, it will be useful to notice that since we consider here the case of low-pressure approximation, when $f(x) \approx f_{c}=$ const, the gradient of the function $f$ is virtually equal to zero along the radius of the configuration. This allows us to expect that the influence of the term $p(\ln f)_{; \nu}$ in Eq. A.2 will be small compared with the gradient term $p_{; \nu}$. Indeed, the numerical calculations show that the smaller the value of the relativistic parameter $\sigma_{s}$, the smaller the value of the term $p(\ln f) ; \nu \operatorname{compared}$ with $p_{; \nu}$. One would expect that in the nonrelativistic limit, when $\sigma_{s} \rightarrow 0$, the extra acceleration associated with the nonminimal coupling will be negligibly small compared with the acceleration provided by a gravitational field.

It is natural to assume that the nonrelativistic approximation used in the present paper is also relevant in describing relatively small objects such as planets. Consistent with this, let us estimate the value of extra acceleration in a nonrelativistic ("Newtonian") system in the presence of a nonminimal coupling such as $p f(\varphi)$. In doing so, we will compare the gravitational acceleration of a fluid element with the extra acceleration associated with the nonminimal coupling. To do this, let us find from Eq. (A.2) the expression for the usual three-acceleration in the nonrelativistic approximation $p \ll \varepsilon$

$$
a_{\mathrm{tot}} \approx a_{N}\left(1+\frac{a_{\mathrm{ch}}}{a_{N}}\right),
$$

where $a_{\text {tot }}$ is the total radial acceleration defined as a sum of the Newtonian acceleration, $a_{N}=-\partial \psi / \partial r(\psi$ is the Newtonian gravitational potential), and the extra acceleration provided by the chameleon, $a_{\mathrm{ch}}=-(p / \rho)(d \ln f / d r)$.

To compare the expression (A.3) with experimental data, it is convenient to use the Eötvös parameter,

$$
\eta \equiv 2 \frac{\left|a_{1}-a_{2}\right|}{\left|a_{1}+a_{2}\right|}
$$

which describes the difference in relative free-fall acceleration for two bodies of different composition having the accelerations $a_{1}$ and $a_{2}$. As mentioned above, $\eta$ is experimentally restricted to be less than $10^{-13}$. Taking into account the smallness of $\eta$, one can find from the above expression:

$$
a_{1} \approx a_{2}(1+\eta)
$$


Compare the expression (A.3) with (A.4): whereas the former gives the magnitude of the total acceleration of a fluid element in the presence of the nonminimal coupling, the latter describes the difference between the accelerations of two test bodies which are manufactured to have different compositions. But in both cases the difference in accelerations is estimated, related to either the nonminimal coupling (in the first case) or a different composition of bodies (in the second case). It is clear that independently of the fact which case is under consideration, the experiment requires that the difference in accelerations be less than $\eta \approx 10^{-13}$. Then, by identifying formally $a_{\text {tot }}=a_{1}, a_{N}=a_{2}$, and $a_{\mathrm{ch}} / a_{N}=\eta$, we have the following restriction on the extra acceleration from (A.3):

$$
\left|a_{\mathrm{ch}}\right| \equiv \frac{p}{\rho} \frac{d \ln f}{d r} \lesssim 10^{-13} a_{N} .
$$

Here the pressure $p$ and the mass density $\rho$ refer to a fluid element falling onto an attractor. The interaction of this matter with a chameleon field through the function $f$ results in the extra acceleration $a_{\mathrm{ch}}$, the value of which must satisfy the condition A.5 to be consistent with the experiment.

It is seen that the condition (A.5) is trivially satisfied if a continuous medium, located in the Earth's gravitational field, is pressureless. Such a case corresponds, for instance, to dustlike matter. If the pressure is nonzero, the problem requires a separate analysis for each particular case. Then, from the point of view of experiment, each element of such a fluid can be considered as a test particle moving in a given gravitational field. In this case the magnitude of extra acceleration will depend on the composition of this medium, which, in turn, is determined by use of various equations of state $p=p(\rho)$ for different forms of matter. Then, aside from the fact that the extra acceleration does take place for one type of matter, there will also be additional differences in accelerations for fluids having different compositions.

\section{Extra accelerations in the Earth's atmosphere}

To illustrate the above considerations, let us now demonstrate how one can estimate the value of extra accelerations, which could appear, for instance, in the Earth's atmosphere. As is known, the Earth's atmosphere is not homogeneous and the air density decreases with altitude; the dependence of the density $\rho$ on altitude $R$ can be approximated by the barometric formula

$$
\rho=\rho_{0} e^{-\left(R-R_{\oplus}\right) / \Delta} .
$$

Here $\rho_{0}$ is the density at sea level, $R=R_{\oplus}$, and $\Delta$ is the so-called scale height of the standard atmosphere, which at the Earth's surface is approximately equal to $8.5 \mathrm{~km}$. For our purposes, we need only to pick this layer of the atmosphere, i.e., in further calculations we assume that $R_{\oplus} \leq R \lesssim\left(R_{\oplus}+\Delta\right)$. This choice of $\rho$ implies that the air temperature does not change with altitude. This allows one to describe the atmosphere by using the equation of state for an isothermal fluid given in the form

$$
p=K \rho
$$

with $K=p_{0} / \rho_{0}=c_{a}^{2}$, where $c_{a}$ is the speed of sound in the air, and $p_{0}$ is the pressure at sea level. Using this equation of state and introducing the dimensionless variables

$$
e^{-\chi}=\frac{\rho}{\rho_{0}}, \quad \zeta=\frac{R}{L}, \quad \phi(\zeta)=\frac{\sqrt{4 \pi G}}{K} \varphi(r),
$$

where $L=\sqrt{K /\left(4 \pi G \rho_{0}\right)}$ has dimensions of length, one can obtain from the Lagrangian (1) the following nonrelativistic equations describing the isothermal fluid in the presence of a chameleon scalar field [19]:

$$
\begin{aligned}
& \frac{1}{\zeta^{2}} \frac{d}{d \zeta}\left(\zeta^{2} \frac{d \chi}{d \zeta}\right)=f e^{-\chi} \\
& \frac{1}{\zeta^{2}} \frac{d}{d \zeta}\left(\zeta^{2} \frac{d \phi}{d \zeta}\right)=-e^{-\chi} \frac{d f}{d \phi} .
\end{aligned}
$$

The first equation is the equation of hydrostatic equilibrium of a fluid in a Newtonian gravitational field. Substituting the given distribution of density (A.6) in (A.9), one can find

$$
f[\phi(\zeta)]=2 \beta \frac{\exp \left[\beta\left(\zeta-\zeta_{\oplus}\right)\right]}{\zeta}
$$


where $\beta=L / \Delta \approx 10^{3}$. Using this expression in A.10 and taking into account that $\beta \gg 1$, one can obtain an equation for the scalar field in the form

$$
\frac{1}{2}\left(\phi^{\prime 2}\right)^{\prime}+\frac{2}{\zeta} \phi^{\prime 2}=-\frac{2 \beta^{2}}{\zeta}
$$

where the prime denotes differentiation with respect to $\zeta$. The first integral of this equation is

$$
\phi^{\prime 2}=\frac{C_{1}}{\zeta^{4}}-\beta^{2}
$$

The value of the integration constant $C_{1}$ is determined from the condition that at the edge of the atmosphere $\phi^{\prime} \approx 0$ (since outside the atmosphere the scalar field makes a smooth transition into the cosmological chameleon field). Representing $\zeta$ in a form $\zeta=\zeta \oplus+\delta$, where $\delta=\Delta / L \approx 10^{-3}$, and substituting this in the above expression, we finally get

$$
\phi^{2}=\beta^{2}\left[\left(\frac{\zeta_{\oplus}}{\zeta}\right)^{4}\left(1+\frac{4 \delta}{\zeta \oplus}\right)-1\right] .
$$

The solution of this equation gives the distribution of the scalar field in the Earth's atmosphere. We will seek a solution starting from the Earth's surface, $\zeta=\zeta_{\oplus}$, and choosing the initial value of $\phi\left(\zeta_{\oplus}\right)$ in such a way as to get approximately the cosmological value of the field at the outer boundary of the atmosphere.

Since the cosmological scalar field, $\phi(\xi)$, and that used here, $\phi(\zeta)$, are measured in different units, then, to find the relation between them, compare their definitions given by the expressions (11) and (A.8), respectively. Thus we have

$$
\phi(\xi)=\sqrt{2} \frac{K}{c^{2}} \phi(\zeta)
$$

Substituting this expression in (21), we get

$$
f=\frac{\Omega_{\Lambda}}{\Omega_{m}}\left[-\frac{\sigma(n+1)}{2}\right]^{n}\left(\sinh \sqrt{\frac{3}{4}} \frac{K}{c^{2}} \phi(\zeta)\right)^{-2(n+1)} .
$$

Next, the experimental restriction from (A.5) on the extra acceleration near the Earth's surface can be rewritten, by using the dimensionless variables from (A.8), as follows:

$$
\left[\frac{d \ln f}{d \zeta}\right]_{\oplus} \lesssim 10^{-10}
$$

Here we have used the following numerical values: $a_{N} \approx 10 \mathrm{~m} \mathrm{~s}^{-2}$ and $K=p_{0} / \rho_{0} \approx 10^{5} \mathrm{~m}^{2} \mathrm{~s}^{-2}$. Then, evaluating $\phi(\zeta)$ numerically by using Eq. A.11), and substituting this in (A.13), it must be checked whether the condition (A.14) is satisfied or not. The calculations indicate that the value of the logarithmic derivative of (A.13),

$$
\frac{d \ln f}{d \zeta}=-\sqrt{3}(n+1) \frac{K}{c^{2}} \phi^{\prime}(\zeta) \operatorname{coth}\left[\sqrt{\frac{3}{4}} \frac{K}{c^{2}} \phi(\zeta)\right],
$$

near the Earth's surface is of order $10^{-10}$ for the values of $n$ used in the present paper. Thus, the condition (A.14) may be considered as satisfied, in principle. The estimate for the condition (A.5) for other forms of matter requires a separate analysis.

[1] V. Sahni, Lect. Notes Phys. 653, 141 (2004).

[2] E. J. Copeland, M. Sami, and S. Tsujikawa, Int. J. Mod. Phys. D 15, 1753 (2006) arXiv:hep-th/0603057.

[3] A. De Felice and S. Tsujikawa, Living Rev. Rel. 13, 3 (2010) arXiv:1002.4928 [gr-qc]].

[4] S. 'i. Nojiri and S. D. Odintsov, Phys. Rep. 505, 59 (2011) arXiv:1011.0544 [gr-qc]].

[5] R. Maartens, Living Rev. Rel. 7, 7 (2004) gr-qc/0312059.

[6] V. Dzhunushaliev, V. Folomeev, and M. Minamitsuji, Rept. Prog. Phys. 73, 066901 (2010) [arXiv:0904.1775 [gr-qc]]. 
[7] J. Khoury and A. Weltman, Phys. Rev. Lett. 93, 171104 (2004). astro-ph/0309300.

[8] J. Khoury and A. Weltman, Phys. Rev. D 69, 044026 (2004) arXiv:astro-ph/0309411.

[9] P. Brax, C. van de Bruck, A. -C. Davis, J. Khoury, and A. Weltman, Phys. Rev. D 70, 123518 (2004). astro-ph/0408415.

[10] D. F. Mota and D. J. Shaw, Phys. Rev. D 75, 063501 (2007) hep-ph/0608078.

[11] H. Farajollahi and A. Salehi, Int. J. Mod. Phys. D 19, 621 (2010) arXiv:1004.3508 [gr-qc]].

[12] F. Cannata and A. Y. Kamenshchik, Int. J. Mod. Phys. D 20, 121 (2011) arXiv:1005.1878 [gr-qc]].

[13] S. Chattopadhyay and U. Debnath, Int. J. Mod. Phys. D 20, 1135 (2011) arXiv:1105.1091 [gr-qc]].

[14] A. B. Henriques, A. R. Liddle, and R. G. Moorhouse, Phys. Lett. B233, 99 (1989).

[15] A. B. Henriques, A. R. Liddle, and R. G. Moorhouse, Nucl. Phys. B337, 737 (1990).

[16] T. D. Lee and Y. Pang, Phys. Rev. D 35, 3678 (1987).

[17] J. P. Crawford and D. Kazanas, Astrophys. J. 701, 1701 (2009).

[18] V. Dzhunushaliev, V. Folomeev, and D. Singleton, Phys. Rev. D 84, 084025 (2011). arXiv:1106.1267 [astro-ph.SR]].

[19] V. Folomeev, Phys. Rev. D 85, 024008 (2012) arXiv:1108.3395 [astro-ph.SR]].

[20] V. Folomeev and D. Singleton, Phys. Rev. D 85, 064045 (2012) arXiv:1112.1786 [astro-ph.SR]].

[21] S. W. Hawking and G.F.R. Ellis, The Large Scale Structure of Spacetime (Cambridge University Press, Cambridge, England, 1973).

[22] K.P. Stanukovich, Sov. Phys. Dokl. 9, 63 (1964).

[23] K.P. Stanukovich, Unsteady Flows of Continuous Medium (Nauka, Moscow, 1971) [in Russian].

[24] O. Bertolami, F. S. N. Lobo, and J. Paramos, Phys. Rev. D 78, 064036 (2008) arXiv:0806.4434 [gr-qc]].

[25] C. M. Muller, Phys. Rev. D 71, 047302 (2005) astro-ph/0410621.

[26] Ya. B. Zel'dovich and I. D. Novikov, Stars and relativity (Dover, New York, 1996).

[27] R. Tooper, Astrophys. J. 140, 434 (1964).

[28] S. Baessler, B. R. Heckel, E. G. Adelberger, J. H. Gundlach, U. Schmidt, and H. E. Swanson, Phys. Rev. Lett. 83, 3585 (1999). 